\title{
Comparative Performance of Medium-Chain-Length Polyhydroxyalkanoates Production Between Pseudomonas Aeruginosa and Pseudomonas Putida
}

\section{Siriorn Boonyawanich}

King Mongkut's Institute of Technology North Bangkok: King Mongkut's University of Technology North Bangkok

Pinanong Tanikkul

Rajamakala University of Technology Rattanakosin: Rajamangala University of Technology Rattanakosin

\section{Pornpanna Thenchartanan}

King Mongkut's Institute of Technology North Bangkok: King Mongkut's University of Technology North Bangkok

Nipon Pisutpaisal ( $\nabla$ nipon.p@sci.kmutnb.ac.th )

King Mongkut's University of Technology North Bangkok https://orcid.org/0000-0003-2605-7139

\section{Research Article}

Keywords: Polyhydroxyalkanoates (PHAs), Pseudomonas putida, Pseudomonas aeruginosa, emulsified palm oil, fatty acid salt

Posted Date: February 10th, 2021

DOI: https://doi.org/10.21203/rs.3.rs-164358/v1

License: (c) (i) This work is licensed under a Creative Commons Attribution 4.0 International License. Read Full License 


\section{Comparative performance of medium-chain-length Polyhydroxyalkanoates production}

2 between Pseudomonas aeruginosa and Pseudomonas putida

3 Siriorn Boonyawanich ${ }^{1,2}$, Pinanong Tanikkul ${ }^{3}$, Pornpanna Thenchartanan ${ }^{1 * *}$, Nipon Pisutpaisal $^{1,2^{*}}$

4

$5 \quad{ }^{1}$ Department of Agro-Industrial, Food, and Environmental Technology (AFET), King Mongkut's

6 University of Technology North Bangkok (KMUTNB)

$7 \quad{ }^{2}$ The Biosensor and Bioelectronics Technology Centre, The Research and Technology Center for

8 Renewable Products and Energy, King Mongkut's University of Technology North Bangkok,

9 Bangkok, 10800, Thailand

$10{ }^{3}$ Department of Environmental Science, Faculty of Science and Technology, Rajamangala University 11 of Technology Rattanakosin, Salaya Campus, 73170, Thailand

* Corresponding author

e-mail: nipon.p@sci.kmutnb.ac.th

** Co- corresponding author

Submit to: Applied Biochemistry and Biotechnology 
Abstract

Non-degradable polymer waste enlarged over increasing of human population. This badly affected on environmental pollution and biosphere changing. Polyhydroxyalkanoates (PHAs), an ecofriendly biopolymer, were substituted for conventional- or petrochemical-derived polymer. They are reserve carbon energy produced from diversity of microorganisms in granular under limited nutrient for bacterial growth (e.g. nitrogen and phosphorus). Pseudomonas aeruginosa TISTR 1287 was used to catalyze emulsified palm oil to produce valuable PHAs. The highest yield of PHAs production $(0.65$ $\mathrm{g} / \mathrm{L}, 38.0 \%$ ) was obtained in MSM supplemented with $0.75 \%$ (v/w) emulsified palm oil after $72-\mathrm{hr}$ cultivation, which was a few lower than that produced by Pseudomonas putida TISTR 1522 (0.95 g/L, $40.15 \%$ ) cultivated in $1 \%(\mathrm{w} / \mathrm{v})$ fatty acid salt for after 24 -hr cultivation. The intracellular PHAs were detected by staining with Nile red. The characters of intracellular PHAs examined by Transmission Electron Microscope (TEM) exhibited that PHAs accumulate in white and roundish-shaped granules with 0.2-0.5 $\square \mathrm{m}$ diameter inside the cells, about 2-3 granules per rod-shaped bacterium cell. These optimizations were successfully demonstrated high content of intracellular PHAs accumulation in $P$. aeruginosa TISTR 1287 by utilization of emulsified palm oil.

Keywords Polyhydroxyalkanoates (PHAs), Pseudomonas putida, Pseudomonas aeruginosa, emulsified palm oil, fatty acid salt

\section{Introduction}

The overpopulation of human has led to the accumulation of enormous amounts of nondegradable petrochemical polymer waste causing environmental pollution and dramatically changing of biosphere [1-3]. To reduce these problems, an environment-friendly polymer was developed to substitute for petrochemical-derived polymers. Polyhydroxyalkanoates (PHAs) are biopolymer produced in granular by a variety of bacteria under unbalance nutrient condition as carbon or reserve material $[1,4-5]$. They have attracted attention as alternative plastic to replace petrochemical polymers with favorable properties of their complete biodegradability and production from a low-cost, renewable feedstock such as palm oil [6-7]. The high carbon content and abundance of palm oil are promising of 
feedstock that can be converted into valuable PHAs by bacteria. Pseudomonas aeruginosa and Pseudomonas putida are promising microorganisms that accumulate high PHAs content and capably utilize a number of feedstock including plant oil [7-10]. The fatty acids in plant oil were favorable carbon source for bacteria growth and catabolized via $\square$-oxidation to produce PHAs $[8,10]$. The PHAs have a broad range of advantageous applications, for instance, a nanoparticle for drug carrier, a part of bioplastic element for molded plastic or single-used polymer, and fertilizer coating film which was applied in agriculture [12].

This study reported the optimization of PHAs production by Pseudomonas aeruginosa TISTR 1287 from emulsified palm oil compared with our previous study by Pseudomonas putida TISTR 1522, lack of secretes lipase ability, from fatty acid salt [7]. The presence and the characters of intracellular PHAs accumulation were analyzed by Nile red staining, fluorescence microscope and Transmission Electron Microscope (TEM).

\section{Materials and methods}

\section{Microorganisms and palm oil}

Pseudomonas aeruginosa TISTR 1287 was obtained by Thailand Institute of Scientific and Technological Research. This pure culture was stored in $30 \%$ glycerol at $-20{ }^{\circ} \mathrm{C}$.

Palm oil (Morakhot olein palm oil), a plant oil, available in local market was used as a sole carbon source for PHAs production. The composition of fatty acid in palm oil was determined as described previously [8]. Gum arabic was discovered as an effective emulsifier for palm oil, with no effect on bacterial growth [10]. In this study, for PHAs production by P. aeruginosa TISTR 1287, bacteria secretes lipase, palm oil was emulsified using Gum arabic as emulsifying agent. Emulsified palm oil was sterilized, mixed with culture medium and adjusted $\mathrm{pH}$ to 6.90 [6, 10, 13]. In comparison to our pervious study, fatty acid salt obtained from saponified palm oil was used as a carbon source to produce PHAs by $P$. putida TISTR 1522, due to its lack of secreted lipase [11]. 


\section{Culture medium and cultivation condition}

Cells were grown in Nutrient Broth (NB) and induced for PHAs production in Mineral Salts Medium (MSM) [9]. The MSM was mixed with emulsified palm from previous experiment before using for PHAs production by $P$. aeruginosa TISTR 1287. Experiments were set up in an orbital shaker incubator $\left(180 \mathrm{rpm}, 30^{\circ} \mathrm{C}\right)$. Cells were harvested by centrifugation at 9,000 rpm for $20 \mathrm{~min}$.

To obtain cell mass for PHAs production, $P$. aeruginosa TISTR 1287 was grown overnight in $50 \mathrm{~mL} \mathrm{NB}$ (first-subculture) before transferring to $500 \mathrm{~mL} \mathrm{NB}$ (second subculture). During lateexponential growth, bacterial cells were harvested by centrifugation and washed with MSM. The standard curve between Colony Forming Unit (CFU) and cell dry weight (CDW) content was used to calculate the amount of initial bacterial cells $\left(10^{11} \mathrm{CFU} \mathrm{mL}-1\right)$ for PHAs production in MSM supplemented with emulsified palm oil [10]. For $P$. putida TISTR 1522, cell mass was obtained as described above.

\section{Optimization for PHAs production}

The influence of various parameters on PHAs production by $P$. aeruginosa TISTR 1287 and $P$. putida TISTR 1522 was studied which are concentration of emulsified palm oil, pH values, CDW content and cultivation time. To optimize concentration of carbon source for PHAs production, bacteria was grown in $300 \mathrm{~mL}$ MSM supplemented with various concentration of emulsified palm oil $(0.50$, $0.75,1.00$ and $1.50 \%(\mathrm{w} / \mathrm{v}))$ for P. aeruginosa TISTR 1287 , and various concentration of fatty acid salt $(0.50,0.75,1.00$ and $1.50 \%(\mathrm{w} / \mathrm{v}))$ for $P$. putida TISTR 1522 . The $\mathrm{pH}$ value of culture medium was measured every $8 \mathrm{hr}$ by $\mathrm{pH}$ meter (Mettler Toledo LE409). To determine CDW content, $2 \mathrm{~mL}$ of culture medium was harvested by centrifugation, washed and dried at $105{ }^{\circ} \mathrm{C}$ for $2 \mathrm{hr}$ [14]. The percentage of emulsified palm oil and fatty acid salt that gave the highest CDW content was selected for the following optimization cultivation time.

\section{PHAs determination}

The accumulation of intracellular PHAs was monitored by fluorescence microscope and Transmission Electron Microscope (TEM). In brief, the aliquot of PHAs-accumulating cell was 
harvested by centrifugation, smeared on glass slide, fixed by heat, stained with $0.05 \mathrm{mM}$ Nile red for 30 min in darkness and analyzed analyzed by Fluorescence Microscope [15]. To study the character of intracellular PHAs, TEM was performed. In brief, PHAs-accumulating cell was collected, washed and stored in $2.5 \%$ glutaraldehyde solution overnight. The resuspension was dehydrated and embedded in BEEM capsule. The polymerized plastic was trimmed, stained with lead citrate followed by $1-2 \%$ uranyl acetate. The sample was washed, dried and analyzed by TEM (TECNAI 20 TWIN) [7].

\section{PHAs extraction}

PHAs extraction was performed as described previously [7]. The PHAs-accumulating cells were harvested, washed with $0.85 \%(\mathrm{w} / \mathrm{v}) \mathrm{NaCl}$ solution and dried at $80{ }^{\circ} \mathrm{C}$ for $5 \mathrm{hr}$. The dried cells $(0.4 \mathrm{~g})$ were soaked in $95 \%$ ethanol, placed in Cellulose Extraction Thimble and extracted by dichloromethane for $5 \mathrm{hr}$ using Sohxlet extractor. The dried weight product (PHAs) was determined after incubation of pellet at $70{ }^{\circ} \mathrm{C}$ for $2 \mathrm{hr}$.

\section{Results and Discussion}

\section{Monitoring of bacterial growth}

In the growth curve of $P$. aeruginosa TISTR 1287 and $P$. putida TISTR 1522, late-exponential growth of bacterial cells exhibited between 10-24 hr and 5-11 hr, respectively (Fig 1a). Moreover, the first- and second-subculture showed both late-exponential growths after $24 \mathrm{hr}$ cultivation for $P$. aeruginosa TISTR 1287, and after18 and $30 \mathrm{hr}$ cultivation for $P$. putida TISTR 1522 (Fig 1b, c). The initial amount of bacterial cells that calculated from standard curve between CFU and CDW content of P. aeruginosa TISTR 1287 and $P$. putida TISTR 1522 (Fig 1d, e) was grown in MSM supplemented of different concentration of emulsified palm oil or fatty acid salt for PHAs production.

Fig. 1 The growth comparison between $P$. aeruginosa TISTR 1287 and P. putida TISTR 1522 from (a) purified stock culture; (b) first-subculture; (c) second-subculture in Nutrient Broth (NB); The linear correlation graph between CFU and CDW content of (d) P. aeruginosa TISTR 1287 and (e) $P$. aeruginosa TISTR 1287 which were used to calculate the amount of initial bacterial cells for PHAs production. 


\section{Influence of carbon source concentration on $\mathrm{pH}$ value and CDW content}

The culture medium of $P$. aeruginosa TISTR 1287 in MSM supplemented with different concentration of emulsified palm oil was taken at interval time to measure the changes of $\mathrm{pH}$ value and CDW content. The result exhibited that $\mathrm{pH}$ value of culture medium of $P$. aeruginosa TISTR 1287 rapidly decreased in the first $12 \mathrm{hr}$ cultivation for all emulsified palm oil concentration and then remained or slightly decreased. On the other hand, previous study reported that the $\mathrm{pH}$ value of culture medium of $P$. putida TISTR 1522 extremely decreased in the first $16 \mathrm{hr}$ cultivation for all carbon source concentration and then slightly increased over cultivation time [7], which showed the $\mathrm{pH}$ values in the ranging of 6.34-6.40 for P. aeruginosa TISTR 1287 and 6.22-6.47 for P. putida TISTR 1522 (Fig 2). The $\mathrm{pH}$ value of culture media decreased in consequence of triglyceride catabolism in palm oil by secreted lipase from P. aeruginosa TISTR 1287, which breaks ester bond of triglycerides into fatty acids and glycerol resulting in lower $\mathrm{pH}$ value in culture medium [16]. Moreover, the variation of $\mathrm{pH}$ value in culture medium depends on types and concentration of carbon sources [17]. for $P$. aeruginosa TISTR 1287 (this study) and $24 \mathrm{hr}$ cultivation for $P$. putida TISTR 1522 [7] which correspond to rapidly decreasing of $\mathrm{pH}$ value of culture medium. The highest $\mathrm{CDW}$ content was 2.33 $\mathrm{g} / \mathrm{L}$ in $0.75 \%(\mathrm{w} / \mathrm{v})$ emulsified palm oil after $44 \mathrm{hr}$ cultivation of $P$. aeruginosa TISTR 1287 and 3.00 $\mathrm{g} / \mathrm{L}$ in $1.00 \%(\mathrm{w} / \mathrm{v}$ ) fatty acid salt after $24 \mathrm{hr}$ cultivation of $P$. putida TISTR 1522 . Afterwards, bacterial growth rate decreased result from continuously decreasing of $\mathrm{pH}$ value which was unsuitable for bacterial growth. This result led to unbalance of hydrogen and hydroxide ions which impact on nutrients feeding of bacterial cell [18]. The observation could be established the correlation between the variation of $\mathrm{pH}$ value of culture medium and $\mathrm{CDW}$ content, and carbon source limitation for bacterial growth and PHAs accumulation which was $0.75 \%$ (w/v) emulsified palm oil for P. aeruginosa TISTR 1287 (this study) and $1.00 \%$ (w/v) fatty acid salt for P. putida TISTR 1522 [7]. Therefore, this emulsified palm oil concentration was selected for further optimization for PHAs production. 
Fig. $2 \mathrm{pH}$ variation of $P$. aeruginosa TISTR 1287 during 72-hr cultivation in MSM supplemented with various concentration of emulsified palm oil.

Fig. 3 CDW variation of $P$. aeruginosa TISTR 1287 during 72-hr cultivation in MSM supplemented with various concentration of emulsified palm oil.

\section{Profile of PHAs production}

The PHAs-accumulating cells were taken at interval time, extracted to measure CDW content and PHAs concentration. The result revealed that PHAs concentration increased over cultivation time up to maximum value at $0.65 \mathrm{~g} / \mathrm{L}$ with CDW content of $1.71 \mathrm{~g} / \mathrm{L}$ and $38.0 \%$ PHAs content after $72 \mathrm{hr}$ cultivation for $P$. aeruginosa TISTR 1287 (Table 1). This observation was noticeable that PHAs accumulation and CDW content increased when the $\mathrm{pH}$ value decreased (Fig 4), consistent with previous result that PHAs production by $P$. putida TISTR 1522 in MSM supplemented with $1 \%$ fatty acid salt which obtained maximum value at $1.23 \mathrm{~g} / \mathrm{L}$ with $\mathrm{CDW}$ content of $3.23 \mathrm{~g} / \mathrm{L}$ after $60 \mathrm{hr}$ cultivation [7]. Accordingly, P. putida KT2442 and P. aeruginosa ATCC 9027 produce PHAs during exponential growth [19-20], and PHAs production relied on the nutrient depletion on medium [6, 9, 11, 13-14]. These result show that emulsified palm oil and fatty acid salt can be a good carbon source for PHAs production using $P$. aeruginosa TISTR 1287 and $P$. putida TISTR 1522. Comparison of our PHAs production yield with that from other reports reveals that our PHAs production from $P$. aeruginosa TISTR 1287 was higher than previously reported data using other $P$. aeruginosa strains, and comparable or higher than previously reported data using other P. putida strains (Table 2). The FTIR result of purified PHAs that produced from P. aeruginosa TISTR 1287 cultivated in $0.75 \%(w / v)$ emulsified palm oil, and NMR result of purified PHAs obtained from P. putida TISTR 1522 cultivated in $1.0 \%(w / v)$ fatty acid salt were characterized as medium-chain length PHAs [7, 27]. These previous results were consistent with previous report that there are 2 major monomer units (medium-chain length) of PHAs production from palm oil which are 3-hydroxyoctanoate (C8) and 3-hydroxydecanoate (C10) [8]. 
Fig. 4 The correlation between PHAs content, CDW content and PHAs concentration of $P$. aeruginosa TISTR 1287 during 60-hr cultivation in MSM supplemented with $0.75 \%$ emulsified palm

oil.

\section{Table 2}

PHAs accumulation analysis by fluorescence microscope and Transmission Electron Microscope

The present of intracellular PHAs was monitored by staining of Nile red on PHAs-accumulating cells and visualizing by fluorescence microscope. An increasing of fluorescence intensity was observed inside bacterial cells which clearly demonstrate $P$. aeruginosa TISTR 1287 accumulated intracellular

Fig. 5 Nile red staining of PHAs accumulation by P. aeruginosa TISTR 1287 in MSM supplemented with $0.75 \%$ (w/v) emulsified palm oil (a) 24-hr (b) 44-hr cultivation visualized by fluorescence 
Fig. 6 The PHAs character accumulating inside P. aeruginosa TISTR 1287 in MSM supplemented with $0.75 \%$ (w/v) emulsified palm oil during 72-hr cultivation under TEM with (a) 2,500x and (b) $5,000 x$ magnification.

\section{CONCLUSION}

Substitution of petrochemical-derived polymer by environmentally degradable biopolymer such as PHAs was developed to reduce environmental pollution. One of the major limitations of the PHAs production in industry is mainly depends on cost of carbon source for bacterial growth. This study demonstrated that a low-cost and abundance palm oil can be a good carbon source for production of valuable PHAs as it can induce the high content of PHAs accumulation using P. aeruginosa TISTR 1287. We also concluded that the culture medium supplemented with $0.75 \%(\mathrm{v} / \mathrm{w})$ emulsified palm oil is the most suitable condition for intracellular PHAs production by P. aeruginosa TISTR 1287 in lab scale. However, it is unlikely to apply in industry owing to cost of gum arabic. Thus development of utilization of low-cost materials could lead to economical and sustainable PHAs manufacturing in the future.

\section{Acknowledgements}

The authors are thankful to the financial support from King Mongkut's University of Technology North Bangkok (KMUTNB-62- KNOW-07), Royal Academy of Engineering (RAEng, UK) and Office of Higher Education Commission (OHEC, Thailand)-Industry Academy Partnership Program (IAPP18-19175).

\section{Ethical Approval}

Our work did not involve human subjects and/or animals.

\section{Consent to Participate}

'Not applicable' 
247 'Not applicable'

\section{Authors Contributions}

250 Conceptualization: Nipon Pisutpaisal and Pinanong Tanikkul; Methodology: Siriorn Boonyawanich, 251 Nipon Pisutpaisal, and Pinanong Tanikkul;; Formal analysis and investigation: Pornpanna 252 Thenchartanan, Nipon Pisutpaisal and Pinanong Tanikkul; Writing - original draft preparation: 253 Pornpanna Thenchartanan, Nipon Pisutpaisal and Pinanong Tanikkul; Writing - review and editing: 254 Nipon Pisutpaisal; Funding acquisition: Nipon Pisutpaisal; Resources: Nipon Pisutpaisal; Supervision: 255 Nipon Pisutpaisal.

256 We confirm that the manuscript has been read and approved by all named authors.

257 We confirm that the order of authors listed in the manuscript has been approved by all named authors.

Funding

260 Funding was received for this work.

261 All of the sources of funding for the work described in this publication are acknowledged below:

262 - King Mongkut's University of Technology North Bangkok (KMUTNB-62- KNOW-07),

263 - Royal Academy of Engineering (RAEng, UK) and Office of Higher Education Commission (OHEC, Thailand)-Industry Academy Partnership Program (IAPP18-19\75)

\section{Competing Interests}

The authors declare that they have no known competing financial interests or personal relationships that could have appeared to influence the work reported in this paper.

\section{Availability of data and materials}

All data generated or analyzed during this study are included in this published article. 
1. Luengo JM, García B, Sandoval A, Naharro G and Olivera ER (2003) Bioplastics from microorganisms. Curr Opin Microbiol 6:251-260

2. Kumar MS, Mudliar SN, Reddy KMK and Chakrabarti T (2004) Production of biodegradable plastics from activated sludge generated from a food processing industrial wastewater treatment plant. Bioresour Technol 95:327-330

3. Divya G, Archana T and Manzano RA (2013) Polyhydroxy Alkanoates - A Sustainable Alternative to Petro-Based Plastics. J Pet Environ Biotechnol 4:1-8. https://doi.org/10.4172/2157-7463.1000143

4. Naik S, Venu Gopal SK and Somal P (2008) Bioproduction of polyhydroxyalkanoates from bacteria: a metabolic approach. World J Microbiol Biotechnol 24:2307-2314

5. Chakraborty P, Gibbons W and Muthukumarappan K (2009) Conversion of volatile fatty acids into polyhydroxyalkanoate by Ralstonia eutropha. J Appl Microbiol 106:1996-2005

6. Follonier S, Panke S and Zinn M (2011) A reduction in growth rate of Pseudomonas putida KT2442 counteracts productivity advances in medium-chain-length polyhydroxyalkanoate production from gluconate. Microb Cell Fact 10:1-11. https://doi.org/10.1186/1475-2859-10-25

7. Boonyawanich S, Tanikkul P, Thenchartanan P, and Pisutpaisal N (2021) Productivity of Pseudomonas putida TISTR 1522 in polyhydroxyalkanoates (PHAs) production from saponified palm oil. Appl. Biochem. Biotechnol. DOI: https://doi.org/10.1007/s12010-020-03481-w.

8. Marsudi S, Unno H and Hori K (2008) Palm oil utilization for the simultaneous production of polyhydroxyalkanoates and rhamnolipids by Pseudomonas aeruginosa. Appl Microbiol Biotechnol 78:955-961.

9. Silva-Queiroz SR, Silva LF, Pradella JGC, Pereira EM and Gomez JGC (2009) PHAMCL biosynthesis systems in Pseudomonas aeruginosa and Pseudomonas putida strains show differences on monomer specificities. J Biotechnol 143:111-118 

kernel oil and its major free fatty acids as carbon substrates for the production of polyhydroxyalkanoates in Pseudomonas putida PGA1. Appl Microbiol Biotechnol 47:207-211

12. Poltronieri P and Kumar P (2017) Polyhydroxyalcanoates (PHAs) in Industrial Applications. In: Martínez LMT (eds) Handbook of Ecomaterial, Springer, Cham, Switzerland, pp. 1-30 polyhydroxyalkanoates from nonanoic acid by co-feeding glucose in carbon-limited, fed-batch culture. J Biotechnol 143:262-267

14. Medjeber N, Abbouni B, Menasria T, Beddal A and Cherif N (2015) Screening and production of polyhydroxyalcanoates by Bacillus megaterium by the using cane and beet molasses as carbon sources.

Der Pharm Lett 7:102-109

15. James BW, Mauchline WS, Dennis PJ, Keevil CW and Wait R (1999) Poly-3-Hydroxybutyrate in Legionella pneumophila, an Energy Source for Survival in Low-Nutrient Environments. Appl Environ Microbiol 65:822-827 Production and characterization of medium-chain-length polyhydroxyalkanoates copolymer from Arctic psychrotrophic bacterium Pseudomonas sp. PAMC 28620. Int J Biol Macromol 97:710-720 17. Grothe E, Moo-Young M and Chisti Y (1999) Fermentation optimization for the production of poly( $\square$-hydroxybutyric acid) microbial thermoplastic. Enzyme Microb Technol 25:132-141

18. Luli GW and Strohl WR (1990) Comparison of Growth, Acetate Production, and Acetate Inhibition of Escherichia coli Strains in Batch and Fed-Batch Fermentations. Appl Environ Microbiol 56:10041011

19. Madison LL and Huisman GW (1999) Metabolic Engineering of Poly(3-Hydroxyalkanoates): From DNA to Plastic. Microbiol Mol Biol Rev 63:21-53

20. Rojas-Rosas O, Villafaña-Rojas J, López-Dellamary FA, Nungaray-Arellano J and GonzálezReynoso O (2007) Production and characterization of polyhydroxyalkanoates in Pseudomonas aeruginosa ATCC 9027 from glucose, an unrelated carbon source. Can J Microbiol 53:840-851 
Polyhydroxyalkanoates by Pseudomonas aeruginosa With Fatty Acids and Alternative Carbon Sources. (2011) Synthesis and characterization of poly(3-hydroxyalkanoates) from Brassica carinata oil with high content of erucic acid and from very long chain fatty acids. Int J Biol Macromol 48:137-145 23. Phukon P, Pokhrel B, Konwar BK and Dolui SK (2012) Biosynthesis and characterization of a new copolymer, poly(3-hydroxyvalerate-co-5-hydroxydecenoate), from Pseudomonas aeruginosa. Biotechnol Lett 35:607-611 Medium-chain-length polyhydroxyalkanoates synthesis by Pseudomonas putida KT2440 relA/ spoT mutant: bioprocess characterization and transcriptome analysis. AMB Expr 7:1-13. https://doi.org/ 10.1186/s13568-017-0396-Z

25. Casini E, de Rijk TC, de Waard P and Eggink G (1997) Synthesis of Poly(hydroxyalkanoate) from Hydrolyzed Linseed Oil. J Environ Polym Degrad 5:153-158 26. Cerrone F, Choudhari SK, Davis R et al (2013) Medium chain length polyhydroxyalkanoate (mclPHA) production from volatile fatty acids derived from the anaerobic digestion of grass. Appl Microbiol Biotechnol 98:611-620

27. Tanikkul P, Sullivan GL Sarp S and Pisutpaisal N (2020) Biosynthesis of medium chain length from

palm

oil.

CSCEE. https://doi.org/10.1016/j.cscee.2020.100045 (2011) Nucleoid-associated PhaF phasin drives intracellular location and segregation of polyhydroxyalkanoate granules in Pseudomonas putida KT2442. Mol Microbiol 79:402-418 29. Tian J, Sinskey AJ and Stubbe J (2005) Kinetic Studies of Polyhydroxybutyrate Granule Formation in Wautersia eutropha H16 by Transmission Electron Microscopy. J Bacteriol 187:3814-3824 

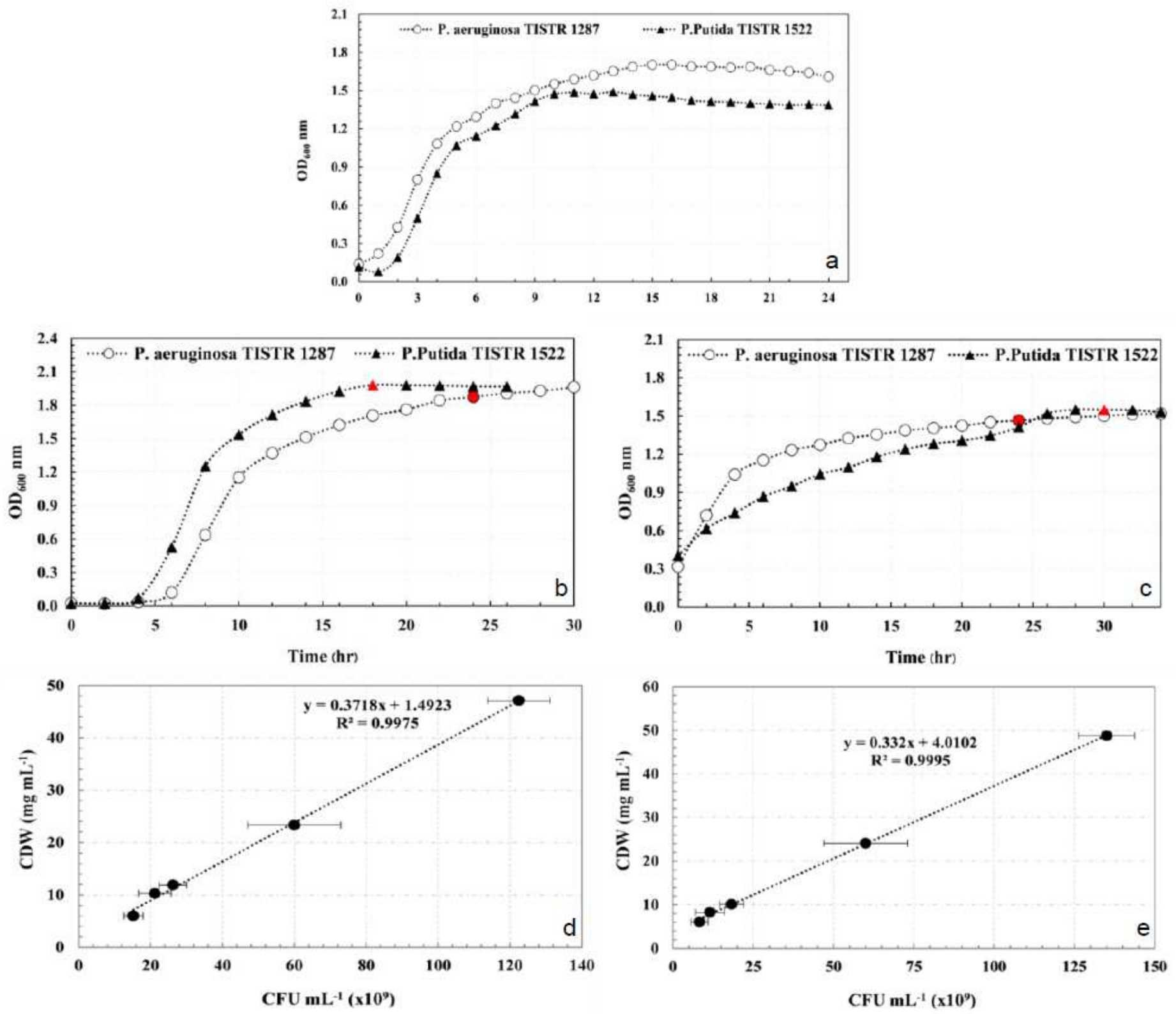

\section{Figure 1}

The growth comparison between P. aeruginosa TISTR 1287 and P. putida TISTR 1522 from (a) purified stock culture; (b) first-subculture; (c) second-subculture in Nutrient Broth (NB); The linear correlation graph between CFU and CDW content of (d) P. aeruginosa TISTR 1287 and (e) P. aeruginosa TISTR 1287 which were used to calculate the amount of initial bacterial cells for PHAs production. 


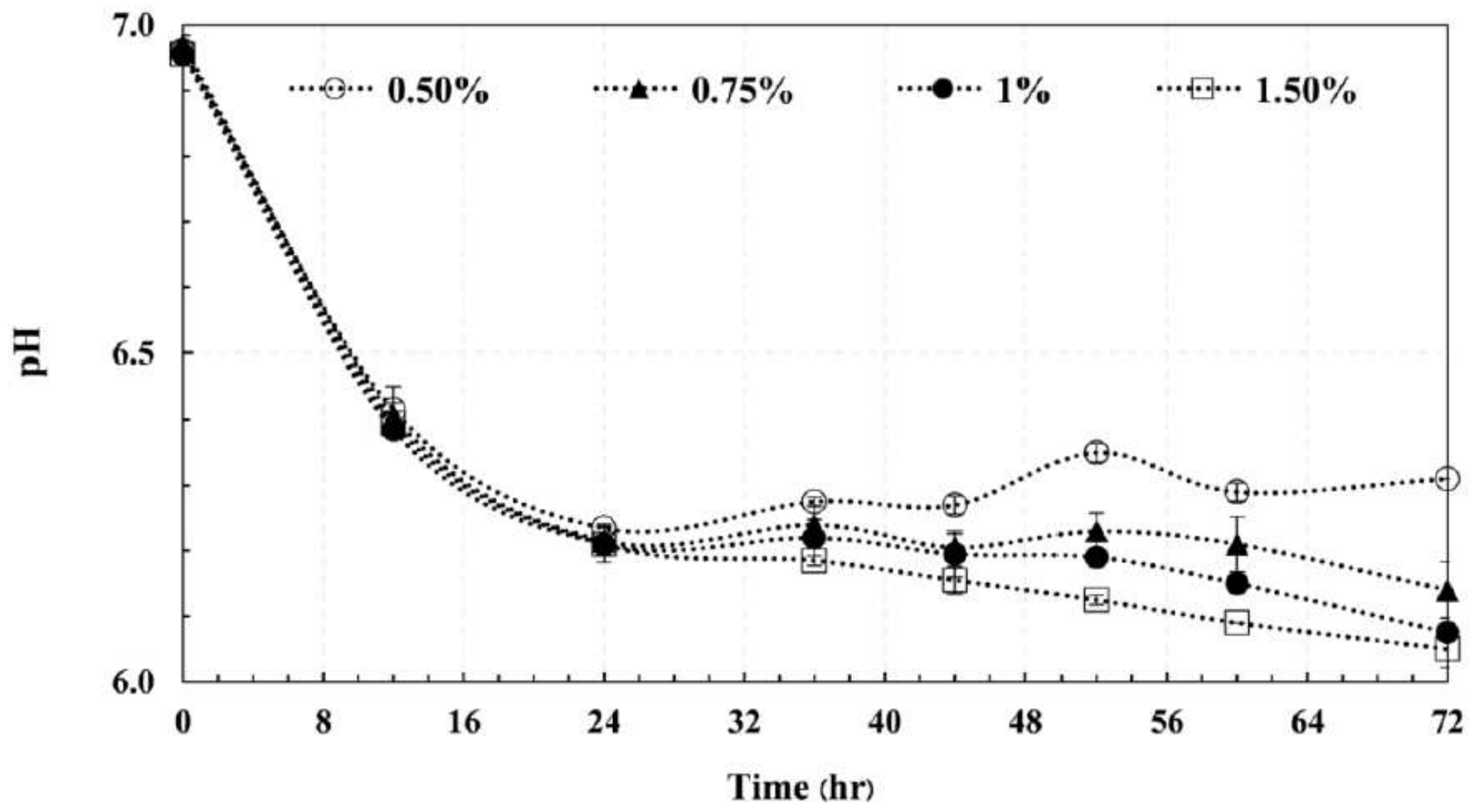

Figure 2

pH variation of P. aeruginosa TISTR 1287 during 72-hr cultivation in MSM supplemented with various concentration of emulsified palm oil.

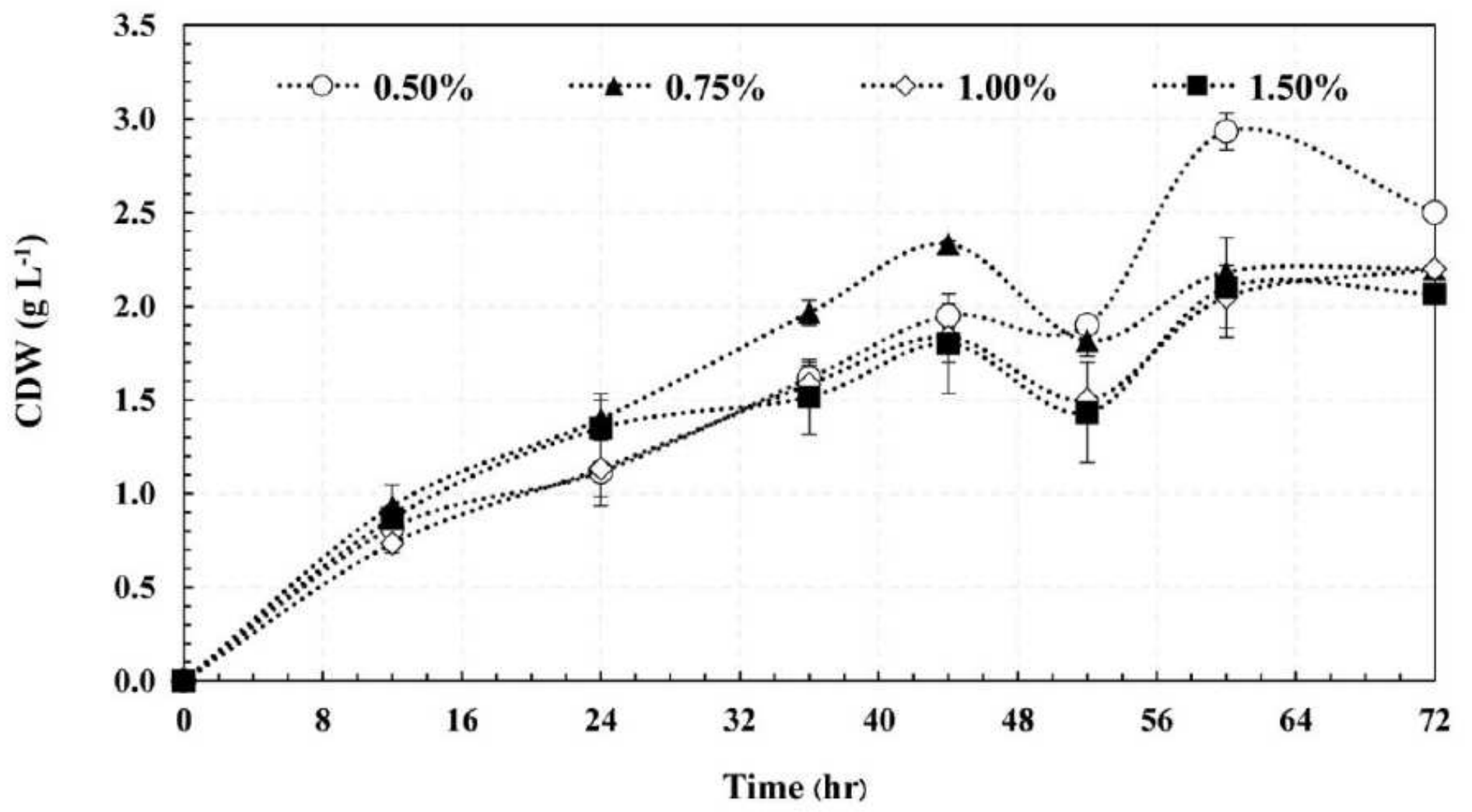




\section{Figure 3}

CDW variation of P. aeruginosa TISTR 1287 during 72-hr cultivation in MSM supplemented with various concentration of emulsified palm oil.

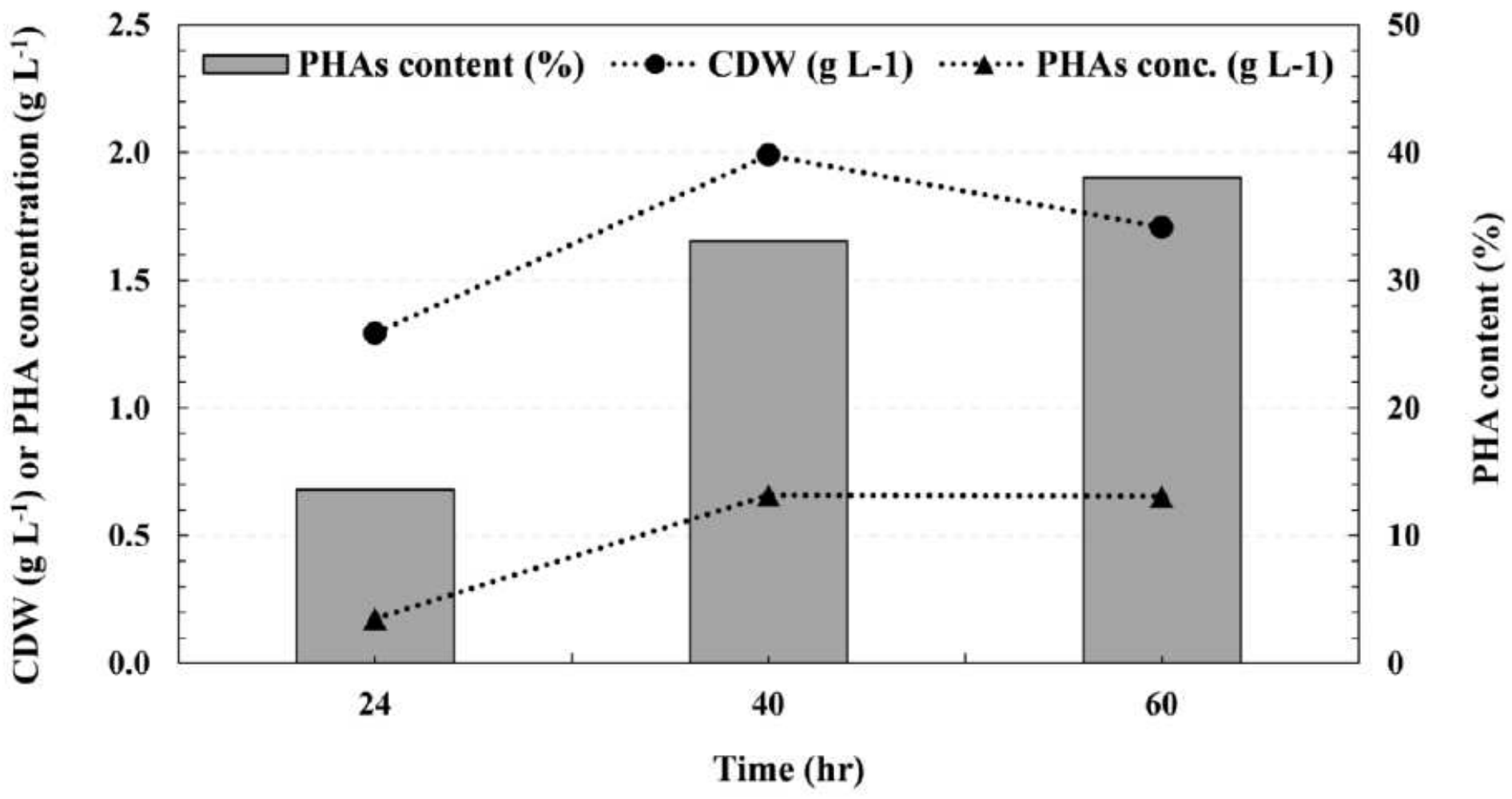

Figure 4

The correlation between PHAs content, CDW content and PHAs concentration of P. aeruginosa TISTR 1287 during 60-hr cultivation in MSM supplemented with $0.75 \%$ emulsified palm oil.

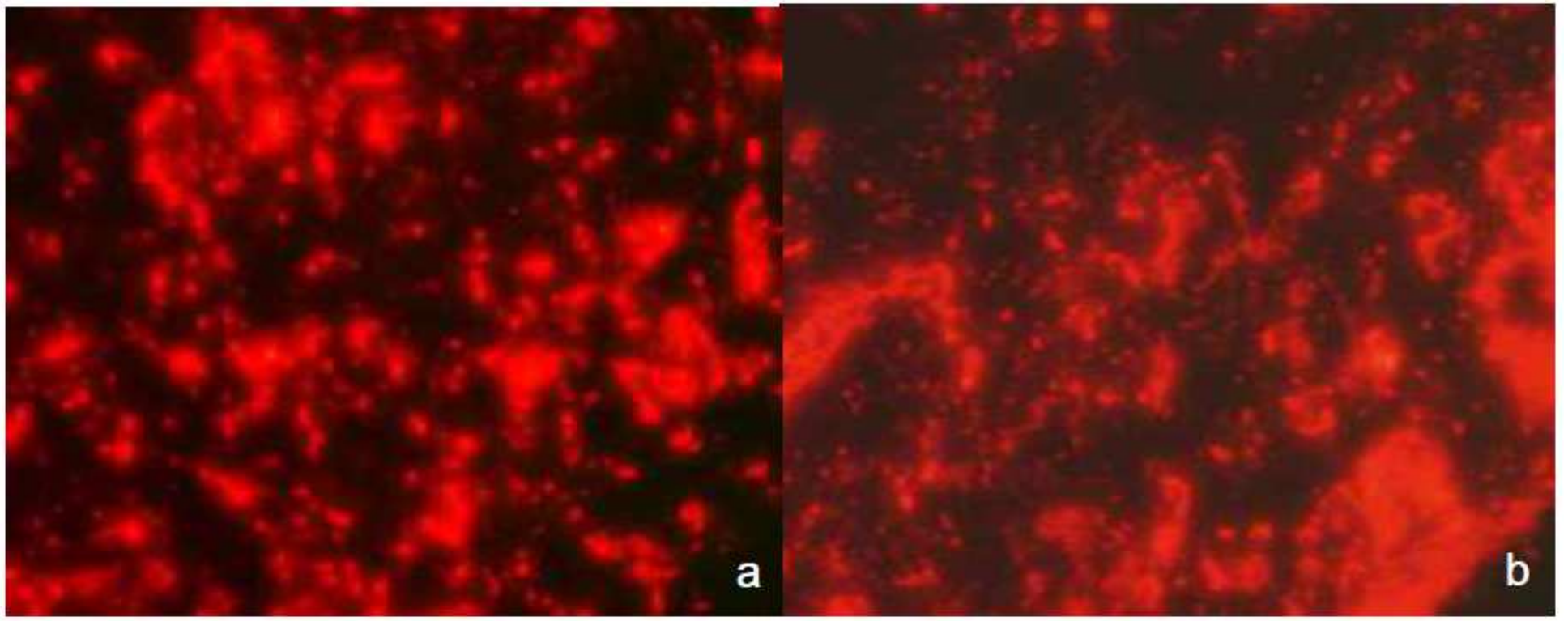

Figure 5 
Nile red staining of PHAs accumulation by P. aeruginosa TISTR 1287 in MSM supplemented with $0.75 \%$ (w/v) emulsified palm oil (a) 24-hr (b) 44-hr cultivation visualized by fluorescence microscope with 400x magnification.

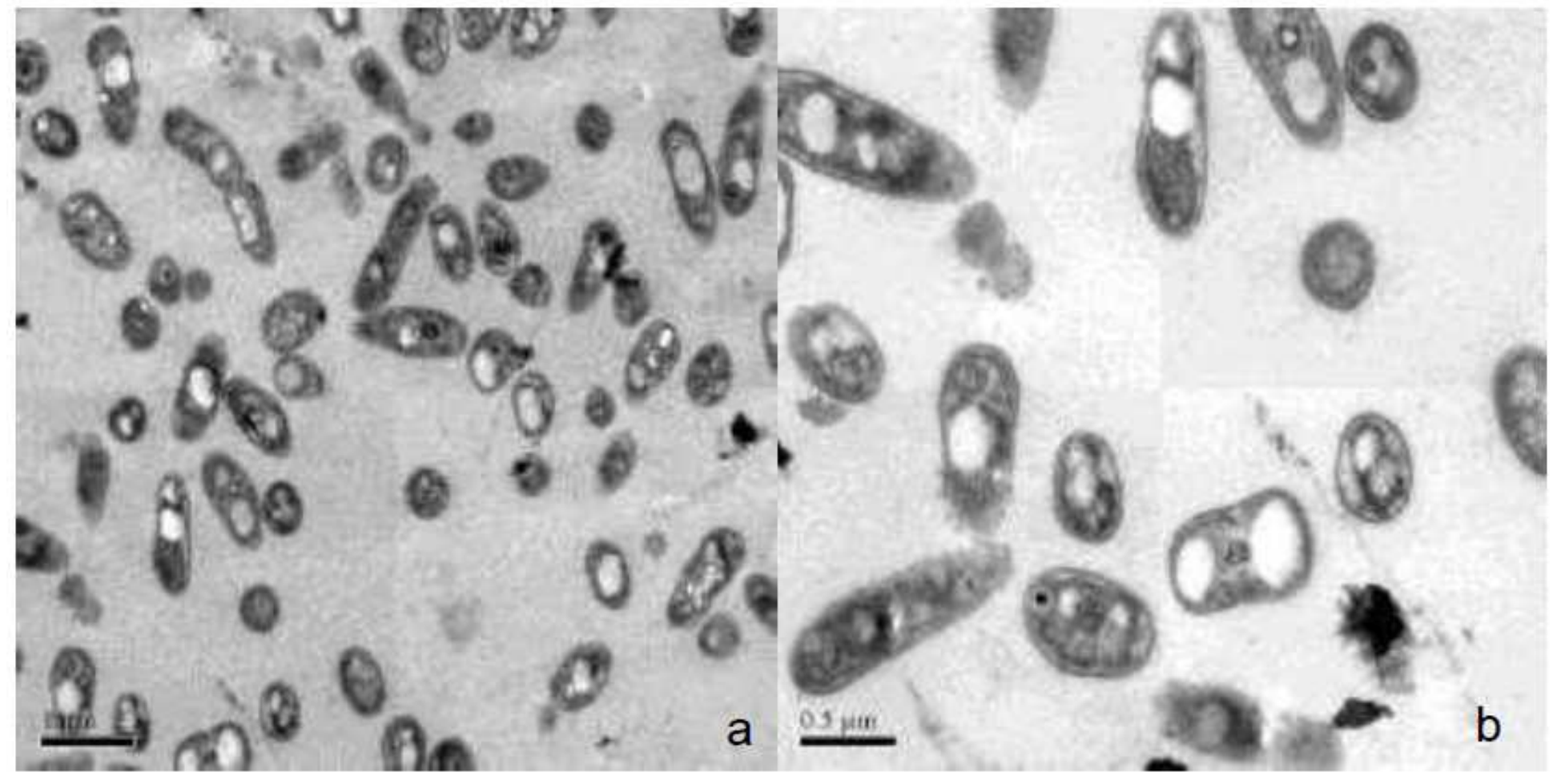

Figure 6

The PHAs character accumulating inside P. aeruginosa TISTR 1287 in MSM supplemented with $0.75 \%$ (w/v) emulsified palm oil during 72-hr cultivation under TEM with (a) 2,500x and (b) 5,000x magnification.

\section{Supplementary Files}

This is a list of supplementary files associated with this preprint. Click to download.

- Table281020.pdf 